\title{
DUS Characterization and Biofortification of Rice Genotypes Derived from Swarna x Ranbir Basmati Cross
}

\author{
N.T. Komala* and R. Gurumurthy \\ College of Agriculture, Dharwad, University of Agricultural Sciences, Dharwad, India \\ *Corresponding author
}

A B S T R A C T

\begin{abstract}
Keywords
Morphological characters, Iron,

Zinc and 160 rice genotypes

Article Info

Accepted:

08 July 2018

Available Online:

10 August 2018

The present investigation was carried out during Kharif 2016 and 2017 at AICRIP, Agricultural Research Station, Mugad, UAS, Dharwad. Among 37 characters studied, the variation was observed in 30 characters viz., coleoptile colour, leaf intensity of green colour, leaf length of blade, leaf width of blade, culm attitude, flag leaf attitude of blade, spikelet density of pubescence of lemma, spikelet colour of stigma, time of heading, stem thickness, stem length, panicle length of main axis, flag leaf attitude of blade, panicle curvature of main axis, panicle number per plant, spikelet colour of tip of lemma, panicle awns, panicle length of longest awn, panicle distribution of awns, panicle attitude of branches, panicle exertion, time maturity, 1000 grains weight, decorticated grain length, decorticated grain width, decorticated grain shape, decorticated grain aroma, iron content and zinc content. There was no distinctness for coleoptile colour, auricles, leaf collar, ligule, shape of ligule, time of heading, presence of secondary branching in panicle, sterile lemma colour and decorticated grain colour, SPAD values, iron and zinc content.
\end{abstract}

\section{Introduction}

Rice belongs to the Poaceae family. The genus Oryza contains 22 species of which only two are cultivated viz., $O$. sativa L. and $O$. glaberrima Steud. Rice is a monocot crop and is normally grown as an annual plant, although in tropical areas it can survive as a perennial and can produce a ratoon crop up to 30 years. The rice plant can grow up to $2.0 \mathrm{~m}$, depending on the variety and soil fertility. It has long slender leaves of 30$100 \mathrm{~cm}$ long and $0.5-2.5 \mathrm{~cm}$ broad. Cultivar characterization is recommended based on the variations in seed and plant morphological characters. In all cultivated crops, including agricultural and horticultural species varietal characterization was attempted by several researchers either for crop registration or to fix their utility in breeding and certification programmes. In a country like India, where contract farming is practiced at many places for seed production, with the active participation of private sector (Mishra et al., 1996), monitoring of genetic purity at each stage of seed production becomes necessary and also cumbersome. In view of this, the study on varietal characterization is highly essential. Plant morphological characters have been recognized as the universally undisputed 
descriptors for DUS testing and varietal characterization of crop varieties. Keeping these facts in view, field experiment were set up for the characterization of several morphological traits helping to develop distinctiveness among the different rice genotypes; to increase the seed yield and nutritional quality of rice.

\section{Materials and Methods}

The experimental material derived from swarna $\times$ Ranbir basmati cross. Swarna is a popular high yielding semi dwarf variety derived from cross between Vasistha $\mathrm{x}$ Mahsuri having low iron and zinc content (2.93 mg $100 \mathrm{~g}^{-1}$ and 2.28mg $100 \mathrm{~g}^{-1}$ respectively). While, Ranbir basmati selection from Basmati 370 possess high iron and zinc content $\left(4.0 \mathrm{mg} 100^{-1} \mathrm{~g}\right.$ and $5.0 \mathrm{mg} 100^{-1} \mathrm{~g}$ respectively). This derived population was used as experimental material in present study to conduct DUS characterization and for estimating seed iron and zinc content during kharif, 2016 and 2017, at All India Coordinate Rice improvement Project (AICRIP), University of Agricultural Sciences, Dharwad. All the agronomic and plant protection measures were adopted as per recommended package of practices for raising healthy crops. The details of parent materials are presented in (Table 1).

\section{Results and Discussion}

Morphological (DUS) characters of rice seedlings derived from Swarna $\times$ Ranbir basmati cross are presented in table 2 . Variations among 160 genotypes were observed for some characters are discussed below. Among 160 genotypes 28 light, 122 medium and 10 genotypes showed dark green colour leaf. Out of 160 genotypes, 61 genotypes were of short, 78 genotypes were medium and 21 genotypes were long leaf length. 119 genotypes were narrow and 41 genotypes were medium of blade width. Similar results obtained by Shrivastava et al., (2015).

Among 160 genotypes, 32 erect, 80 semierect, 46 were open and only two genotypes showing spreading type of clum attitude. Out of 160 genotypes, 51 erect, 78 of semi erect, and 31 with horizontal flag leaf attitude of blade. Pubescence on lemma was absent in 29 genotypes, 125 showed weak pubescence, medium pubescence in five genotypes and one genotype with strong pubescence. Out of 160 genotypes, 110 genotypes had white coloured stigma, two with light green colour, 47 yellow and one genotype with light purple stigma colour.

Table.1 Details of the parents used in the presents study

\begin{tabular}{|l|l|l|c|c|c|c|c|}
\hline \multicolumn{1}{|c|}{ Genotypes } & \multicolumn{1}{|c|}{ Parentage } & $\begin{array}{c}\text { Seed } \\
\text { Type }\end{array}$ & $\begin{array}{c}50 \% \\
\text { Flowering }\end{array}$ & $\begin{array}{c}\text { Iron } \\
\text { content } \\
(\mathrm{mg} / \mathrm{kg} \text { of } \\
\text { brown } \\
\text { rice })\end{array}$ & $\begin{array}{c}\text { zinc } \\
\text { content } \\
(\mathrm{mg} / \mathrm{kg} \text { of } \\
\text { brown } \\
\text { rice })\end{array}$ & $\begin{array}{c}\text { Yield } \\
\left(\mathrm{t} \text { ha }^{-1}\right)\end{array}$ & $\begin{array}{c}\text { Year of } \\
\text { release }\end{array}$ \\
\hline $\begin{array}{l}\text { Swarna } \\
\text { (IET 7041) }\end{array}$ & Vasisa X Mahsuri & MS & 125 & 7.8 & 22.8 & 3.5 & 1979 \\
\hline $\begin{array}{l}\text { Ranbir } \\
\text { Basmati } \\
\text { (IET 11348) }\end{array}$ & $\begin{array}{l}\text { Selection from } \\
\text { Basmati 370 }\end{array}$ & LS & 95 & 13.3 & 29.6 & 2.7 & 1994 \\
\hline
\end{tabular}


Table.2 Morphological (DUS) characters of rice seedlings derived from Swarna $\times$ Ranbir basmati

\begin{tabular}{|c|c|c|c|c|c|c|c|c|c|c|c|c|c|c|c|c|c|c|c|c|c|c|c|c|c|c|c|c|c|c|c|c|c|c|c|c|c|}
\hline $\begin{array}{l}\text { م. } \\
\text { : } \\
\text { பू. }\end{array}$ & $\begin{array}{l}\frac{\ddot{0}}{0} \\
\frac{\tilde{0}}{0} \\
\frac{0}{0} \\
\dot{0}\end{array}$ & 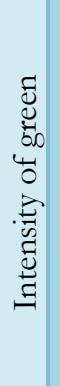 & 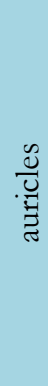 & $\frac{\text { t] }}{\overline{0}}$ & $\stackrel{\vec{v}}{\vec{E}_{0}}$ & 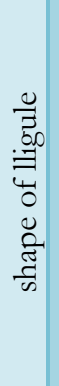 & 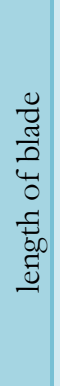 & 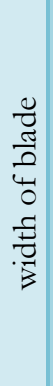 & 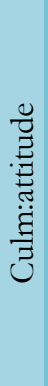 & 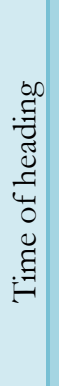 & 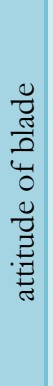 & 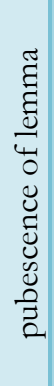 & 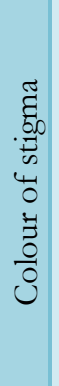 & 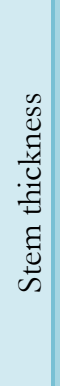 & 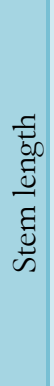 & 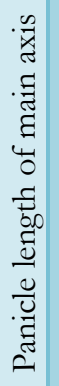 & 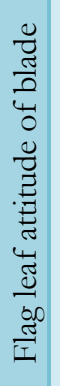 & 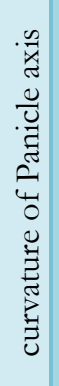 & 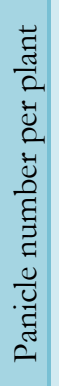 & 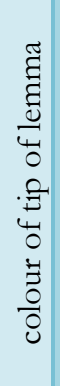 & 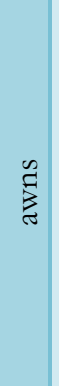 & 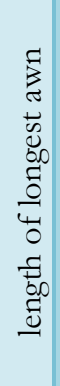 & 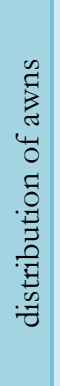 & 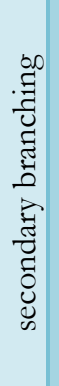 & 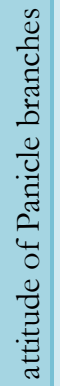 & 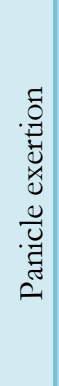 & 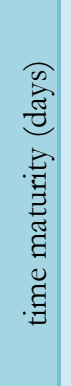 & 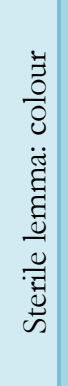 & 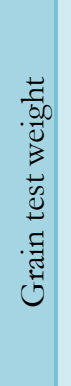 & 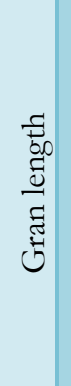 & 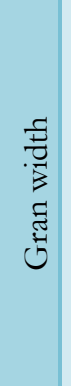 & 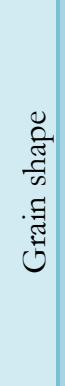 & 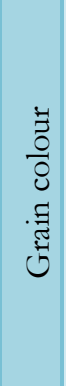 & 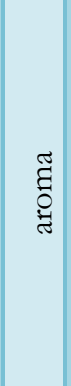 & $\begin{array}{l}\hat{z} \\
\text { के }\end{array}$ & 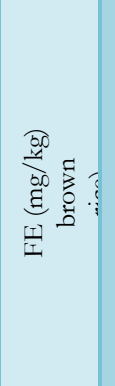 & 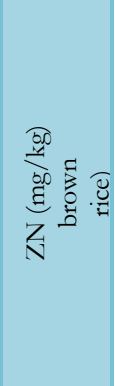 \\
\hline SRSG -1 & 2 & 5 & 9 & 9 & 9 & 3 & 3 & 3 & 5 & 9 & 3 & 1 & 1 & 5 & 7 & 7 & 3 & 3 & 3 & 3 & 1 & - & - & 9 & 5 & 7 & 3 & 1 & 3 & 3 & 3 & 3 & 1 & 1 & 37 & 11.6 & 20.2 \\
\hline SRSG - 2 & 2 & 3 & 9 & 9 & 9 & 3 & 5 & 3 & 1 & 9 & 1 & 3 & 5 & 7 & 1 & 5 & 3 & 3 & 3 & 3 & 9 & 5 & 5 & 9 & 5 & 5 & 5 & 1 & 3 & 3 & 5 & 3 & 1 & 1 & 18 & 14.8 & 26.2 \\
\hline SRSG - 3 & 2 & 5 & 9 & 9 & 9 & 3 & 7 & 3 & 1 & 9 & 3 & 1 & 1 & 5 & 3 & 3 & 3 & 3 & 3 & 3 & 9 & 5 & 5 & 9 & 3 & 3 & 5 & 1 & 5 & 3 & 3 & 3 & 1 & 1 & 25 & 11.8 & 24.0 \\
\hline SRSG - 4 & 2 & 5 & 9 & 9 & 9 & 3 & 5 & 3 & 1 & 9 & 1 & 1 & 5 & 3 & 1 & 5 & 3 & 3 & 3 & 3 & 1 & - & - & 9 & 3 & 3 & 5 & 1 & 3 & 5 & 3 & 5 & 1 & 1 & 27 & 11.3 & 23.1 \\
\hline SRSG - 5 & 2 & 5 & 9 & 9 & 9 & 3 & 5 & 3 & 1 & 9 & 3 & 3 & 5 & 5 & 1 & 3 & 3 & 3 & 3 & 3 & 1 & - & - & 9 & 3 & 3 & 7 & 1 & 3 & 3 & 3 & 3 & 1 & 1 & 27 & 12.2 & 26.4 \\
\hline SRSG - 6 & 2 & 5 & 9 & 9 & 9 & 3 & 5 & 3 & 5 & 9 & 1 & 3 & 1 & 5 & 3 & 5 & 3 & 3 & 3 & 3 & 9 & 1 & 1 & 9 & 1 & 3 & 5 & 1 & 3 & 3 & 3 & 3 & 1 & 1 & 26 & 11.2 & 23.2 \\
\hline SRSG - 7 & 2 & 5 & 9 & 9 & 9 & 3 & 7 & 3 & 3 & 9 & 1 & 3 & 5 & 3 & 5 & 3 & 1 & 1 & 3 & 3 & 1 & - & - & 9 & 3 & 3 & 7 & 1 & 3 & 3 & 3 & 3 & 1 & 1 & 26 & 14.1 & 23.6 \\
\hline SRSG - 8 & 2 & 5 & 9 & 9 & 9 & 3 & 5 & 3 & 5 & 9 & 3 & 1 & 5 & 5 & 5 & 3 & 3 & 3 & 3 & 3 & 1 & - & - & 9 & 3 & 3 & 5 & 1 & 3 & 3 & 3 & 3 & 1 & 1 & 37 & 10.2 & 22.8 \\
\hline SRSG - 9 & 2 & 7 & 9 & 9 & 9 & 3 & 3 & 3 & 5 & 9 & 3 & 1 & 1 & 5 & 1 & 3 & 1 & 3 & 5 & 3 & 1 & - & - & 9 & 3 & 3 & 7 & 1 & 3 & 5 & 3 & 5 & 1 & 1 & 40 & 12.3 & 26.6 \\
\hline SRSG - 11 & 2 & 5 & 9 & 9 & 9 & 3 & 7 & 3 & 3 & 9 & 5 & 3 & 5 & 5 & 7 & 7 & 5 & 3 & 5 & 3 & 1 & - & - & 9 & 3 & 3 & 7 & 1 & 3 & 3 & 3 & 3 & 1 & 1 & 32 & 11.8 & 21.3 \\
\hline SRSG - 12 & 2 & 5 & 9 & 9 & 9 & 3 & 3 & 5 & 3 & 9 & 1 & 1 & 1 & 3 & 1 & 3 & 3 & 3 & 3 & 3 & 9 & 1 & 1 & 9 & 3 & 3 & 5 & 1 & 5 & 3 & 3 & 3 & 1 & 1 & 26 & 13.3 & 25.3 \\
\hline SRSG - 13 & 2 & 5 & 9 & 9 & 9 & 3 & 5 & 3 & 3 & 9 & 1 & 1 & 1 & 5 & 5 & 3 & 3 & 3 & 3 & 3 & 1 & - & - & 9 & 3 & 3 & 7 & 1 & 5 & 5 & 3 & 5 & 1 & 1 & 30 & 17.3 & 24.5 \\
\hline SRSG - 14 & 2 & 5 & 9 & 9 & 9 & 3 & 7 & 3 & 5 & 9 & 1 & 3 & 5 & 5 & 1 & 5 & 5 & 3 & 3 & 3 & 1 & - & - & 9 & 3 & 3 & 7 & 1 & 5 & 3 & 3 & 3 & 1 & 1 & 31 & 15.3 & 30.1 \\
\hline SRSG - 15 & 2 & 5 & 9 & 9 & 9 & 3 & 5 & 5 & 5 & 9 & 3 & 3 & 5 & 5 & 1 & 5 & 5 & 3 & 3 & 3 & 1 & - & - & 9 & 3 & 3 & 7 & 1 & 3 & 3 & 5 & 3 & 1 & 1 & 30 & 13.9 & 26.6 \\
\hline SRSG - 16 & 2 & 5 & 9 & 9 & 9 & 3 & 7 & 5 & 5 & 9 & 5 & 3 & 5 & 7 & 5 & 5 & 5 & 3 & 3 & 5 & 1 & - & - & 9 & 3 & 3 & 7 & 1 & 5 & 3 & 3 & 3 & 1 & 1 & 33 & 13.6 & 27.1 \\
\hline SRSG - 17 & 2 & 5 & 9 & 9 & 9 & 3 & 5 & 5 & 1 & 9 & 3 & 1 & 5 & 5 & 5 & 7 & 3 & 3 & 3 & 3 & 1 & - & - & 9 & 3 & 3 & 5 & 1 & 5 & 3 & 3 & 3 & 1 & 1 & 32 & 12.6 & 21.9 \\
\hline SRSG - 18 & 2 & 5 & 9 & 9 & 9 & 3 & 3 & 3 & 3 & 9 & 1 & 3 & 1 & 3 & 1 & 1 & 3 & 3 & 3 & 3 & 9 & 1 & 1 & 9 & 5 & 3 & 7 & 1 & 3 & 5 & 3 & 5 & 1 & 1 & 33 & 10.8 & 21.2 \\
\hline SRSG - 19 & 2 & 5 & 9 & 9 & 9 & 3 & 3 & 3 & 3 & 9 & 1 & 3 & 1 & 3 & 1 & 1 & 3 & 3 & 3 & 3 & 9 & 1 & 1 & 9 & 3 & 3 & 7 & 1 & 3 & 3 & 3 & 3 & 1 & 1 & 32 & 16.5 & 32.0 \\
\hline SRSG - 20 & 2 & 7 & 9 & 9 & 9 & 3 & 5 & 5 & 5 & 9 & 3 & 3 & 1 & 5 & 3 & 3 & 5 & 3 & 3 & 3 & 9 & 1 & 1 & 9 & 3 & 7 & 7 & 1 & 3 & 3 & 5 & 3 & 1 & 1 & 38 & 10.7 & 23.4 \\
\hline SRSG - 21 & 2 & 7 & 9 & 9 & 9 & 3 & 3 & 3 & 3 & 9 & 5 & 3 & 1 & 3 & 3 & 5 & 3 & 3 & 3 & 5 & 9 & 1 & 1 & 9 & 3 & 3 & 7 & 1 & 3 & 3 & 5 & 3 & 1 & 1 & 40 & 13.2 & 22.2 \\
\hline SRSG - 22 & 2 & 7 & 9 & 9 & 9 & 3 & 3 & 3 & 5 & 9 & 3 & 3 & 1 & 3 & 1 & 5 & 5 & 3 & 3 & 3 & 1 & - & - & 9 & 3 & 3 & 7 & 1 & 5 & 3 & 3 & 3 & 1 & 9 & 36 & 12.5 & 30.2 \\
\hline SRSG - 23 & 2 & 7 & 9 & 9 & 9 & 3 & 3 & 3 & 5 & 9 & 3 & 3 & 1 & 5 & 1 & 5 & 3 & 3 & 3 & 3 & 1 & - & - & 9 & 3 & 3 & 5 & 1 & 3 & 3 & 3 & 3 & 1 & 9 & 35 & 15.2 & 32.7 \\
\hline SRSG - 24 & 2 & 7 & 9 & 9 & 9 & 3 & 5 & 5 & 5 & 9 & 3 & 3 & 1 & 5 & 1 & 5 & 3 & 3 & 3 & 3 & 1 & - & - & 9 & 3 & 3 & 7 & 1 & 3 & 1 & 3 & 2 & 1 & 1 & 35 & 12.3 & 31.6 \\
\hline SRSG - 25 & 2 & 5 & 9 & 9 & 9 & 3 & 5 & 5 & 5 & 9 & 3 & 1 & 5 & 3 & 3 & 5 & 3 & 3 & 3 & 3 & 9 & 3 & 1 & 9 & 3 & 3 & 5 & 1 & 1 & 5 & 5 & 5 & 1 & 1 & 29 & 15.6 & 34.9 \\
\hline SRSG - 26 & 2 & 5 & 9 & 9 & 9 & 3 & 5 & 5 & 5 & 9 & 3 & 3 & 1 & 5 & 3 & 1 & 5 & 5 & 5 & 3 & 1 & - & - & 9 & 5 & 5 & 7 & 1 & 5 & 1 & 5 & 2 & 1 & 1 & 35 & 12.3 & 28.4 \\
\hline SRSG - 27 & 2 & 5 & 9 & 9 & 9 & 3 & 5 & 5 & 3 & 9 & 1 & 1 & 5 & 5 & 1 & 3 & 1 & 3 & 3 & 5 & 9 & 3 & 1 & 9 & 3 & 3 & 5 & 1 & 3 & 3 & 3 & 2 & 1 & 1 & 31 & 12.0 & 16.1 \\
\hline SRSG - 28 & 2 & 5 & 9 & 9 & 9 & 3 & 5 & 5 & 3 & 9 & 1 & 1 & 5 & 5 & 1 & 3 & 1 & 3 & 3 & 3 & 1 & - & - & 9 & 3 & 3 & 5 & 1 & 5 & 3 & 3 & 2 & 1 & 1 & 36 & 14.4 & 26.8 \\
\hline SRSG - 29 & 2 & 5 & 9 & 9 & 9 & 3 & 3 & 3 & 5 & 9 & 1 & 3 & 1 & 3 & 1 & 1 & 1 & 3 & 5 & 3 & 1 & - & - & 9 & 3 & 3 & 5 & 1 & 1 & 1 & 3 & 1 & 1 & 1 & 30 & 15.7 & 25.8 \\
\hline
\end{tabular}




\begin{tabular}{|c|c|c|c|c|c|c|c|c|c|c|c|c|c|c|c|c|c|c|c|c|c|c|c|c|c|c|c|c|c|c|c|c|c|c|c|c|c|}
\hline SRSG - 33 & 2 & 5 & 9 & 9 & 9 & 3 & 5 & 3 & 3 & 9 & 1 & 3 & 1 & 3 & 3 & 3 & 3 & 3 & 3 & 3 & 1 & - & - & 9 & 3 & 3 & 7 & 1 & 5 & 3 & 3 & 3 & 1 & 1 & 30 & 15.8 & 27.3 \\
\hline SRSG - 34 & 2 & 5 & 9 & 9 & 9 & 3 & 3 & 5 & 5 & 9 & 1 & 1 & 5 & 3 & 1 & 3 & 3 & 3 & 3 & 3 & 1 & - & - & 9 & 3 & 3 & 5 & 1 & 3 & 3 & 3 & 3 & 1 & 1 & 36 & 14.1 & 31.7 \\
\hline SRSG - 35 & 2 & 5 & 9 & 9 & 9 & 3 & 3 & 3 & 3 & 9 & 5 & 7 & 1 & 7 & 1 & 3 & 1 & 3 & 5 & 3 & 1 & - & - & 9 & 3 & 3 & 5 & 1 & 1 & 3 & 3 & 3 & 1 & 1 & 31 & 10.5 & 22.8 \\
\hline SRSG - 36 & 2 & 5 & 9 & 9 & 9 & 3 & 5 & 3 & 5 & 9 & 3 & 3 & 1 & 3 & 1 & 3 & 1 & 5 & 3 & 3 & 1 & - & - & 9 & 3 & 7 & 7 & 1 & 1 & 3 & 3 & 2 & 1 & 1 & 31 & 13.5 & 28.5 \\
\hline SRSG - 37 & 2 & 5 & 9 & 9 & 9 & 3 & 3 & 5 & 5 & 9 & 3 & 3 & 1 & 5 & 1 & 1 & 1 & 3 & 7 & 3 & 1 & - & - & 9 & 3 & 3 & 5 & 1 & 3 & 5 & 3 & 5 & 1 & 9 & 37 & 14.8 & 27.4 \\
\hline SRSG - 39 & 2 & 5 & 9 & 9 & 9 & 3 & 3 & 5 & 5 & 9 & 3 & 3 & 1 & 3 & 1 & 3 & 1 & 3 & 5 & 3 & 1 & - & - & 9 & 3 & 3 & 5 & 1 & 1 & 3 & 5 & 3 & 1 & 1 & 35 & 14.4 & 23.0 \\
\hline SRSG - 40 & 2 & 7 & 9 & 9 & 9 & 3 & 5 & 5 & 5 & 9 & 1 & 1 & 1 & 3 & 1 & 3 & 3 & 3 & 5 & 3 & 1 & - & - & 9 & 3 & 3 & 5 & 1 & 3 & 3 & 3 & 3 & 1 & 1 & 45 & 14.1 & 34.8 \\
\hline SRSG - 41 & 2 & 5 & 9 & 9 & 9 & 3 & 7 & 3 & 5 & 9 & 3 & 3 & 5 & 7 & 5 & 5 & 5 & 3 & 3 & 5 & 1 & - & - & 9 & 3 & 5 & 7 & 1 & 5 & 3 & 3 & 3 & 1 & 1 & 34 & 11.1 & 21.3 \\
\hline SRSG - 42 & 2 & 5 & 9 & 9 & 9 & 3 & 7 & 5 & 5 & 9 & 3 & 3 & 1 & 5 & 5 & 3 & 3 & 3 & 5 & 3 & 1 & - & - & 9 & 3 & 3 & 5 & 1 & 3 & 5 & 3 & 5 & 1 & 1 & 32 & 14.9 & 30.8 \\
\hline SRSG - 44 & 2 & 5 & 9 & 9 & 9 & 3 & 3 & 3 & 3 & 9 & 3 & 3 & 5 & 7 & 3 & 3 & 3 & 3 & 3 & 5 & 1 & - & - & 9 & 3 & 5 & 5 & 1 & 5 & 5 & 3 & 5 & 1 & 9 & 33 & 11.9 & 23.1 \\
\hline SRSG - 45 & 2 & 5 & 9 & 9 & 9 & 3 & 3 & 3 & 3 & 9 & 1 & 1 & 1 & 7 & 3 & 1 & 3 & 3 & 3 & 3 & 1 & - & - & 9 & 3 & 3 & 5 & 1 & 3 & 3 & 5 & 3 & 1 & 9 & 29 & 17.0 & 26.3 \\
\hline SRSG - 46 & 2 & 7 & 9 & 9 & 9 & 3 & 3 & 3 & 5 & 9 & 1 & 3 & 5 & 5 & 1 & 3 & 1 & 1 & 3 & 3 & 1 & - & - & 9 & 3 & 3 & 5 & 1 & 3 & 3 & 3 & 3 & 1 & 1 & 43 & 14.8 & 27.3 \\
\hline SRSG - 47 & 2 & 5 & 9 & 9 & 9 & 3 & 7 & 5 & 5 & 9 & 3 & 1 & 1 & 7 & 5 & 5 & 3 & 3 & 3 & 3 & 1 & - & - & 9 & 3 & 5 & 5 & 1 & 7 & 3 & 3 & 3 & 1 & 1 & 27 & 13.7 & 21.8 \\
\hline SRSG - 48 & 2 & 5 & 9 & 9 & 9 & 3 & 7 & 5 & 3 & 9 & 1 & 3 & 5 & 5 & 7 & 5 & 3 & 3 & 3 & 5 & 1 & - & - & 9 & 3 & 5 & 7 & 1 & 5 & 3 & 5 & 3 & 1 & 1 & 35 & 14.5 & 26.0 \\
\hline SRSG - 49 & 2 & 5 & 9 & 9 & 9 & 3 & 5 & 5 & 3 & 9 & 1 & 3 & 1 & 3 & 1 & 5 & 3 & 3 & 3 & 3 & 9 & 1 & 1 & 9 & 3 & 5 & 5 & 1 & 5 & 3 & 3 & 3 & 1 & 1 & 31 & 13.4 & 32.0 \\
\hline SRSG - 50 & 2 & 5 & 9 & 9 & 9 & 3 & 5 & 5 & 5 & 9 & 3 & 3 & 1 & 5 & 5 & 5 & 3 & 3 & 5 & 3 & 1 & - & - & 9 & 3 & 5 & 5 & 1 & 3 & 1 & 3 & 1 & 1 & 1 & 31 & 16.9 & 41.9 \\
\hline SRSG - 51 & 2 & 5 & 9 & 9 & 9 & 3 & 5 & 5 & 3 & 9 & 3 & 1 & 1 & 3 & 5 & 5 & 3 & 3 & 3 & 3 & 9 & 1 & 1 & 9 & 3 & 5 & 7 & 1 & 5 & 5 & 3 & 5 & 1 & 9 & 22 & 13.7 & 29.4 \\
\hline SRSG - 52 & 2 & 5 & 9 & 9 & 9 & 3 & 5 & 5 & 5 & 9 & 3 & 3 & 5 & 5 & 7 & 5 & 3 & 3 & 3 & 3 & 1 & - & - & 9 & 3 & 3 & 7 & 1 & 3 & 3 & 3 & 3 & 1 & 1 & 31 & 13.4 & 23.8 \\
\hline SRSG - 54 & 2 & 5 & 9 & 9 & 9 & 3 & 5 & 5 & 3 & 9 & 5 & 1 & 1 & 5 & 3 & 5 & 3 & 3 & 3 & 3 & 1 & - & - & 9 & 3 & 5 & 5 & 1 & 5 & 3 & 5 & 3 & 1 & 1 & 36 & 13.0 & 22.0 \\
\hline SRSG - 55 & 2 & 5 & 9 & 9 & 9 & 3 & 3 & 3 & 3 & 9 & 3 & 3 & 1 & 5 & 3 & 5 & 3 & 3 & 3 & 3 & 1 & - & - & 9 & 3 & 5 & 5 & 1 & 3 & 3 & 3 & 3 & 1 & 1 & 30 & 13.8 & 27.3 \\
\hline SRSG - 56 & 2 & 5 & 9 & 9 & 9 & 3 & 5 & 3 & 5 & 9 & 1 & 3 & 1 & 5 & 3 & 3 & 5 & 3 & 3 & 3 & 1 & - & - & 9 & 3 & 3 & 5 & 1 & 5 & 1 & 3 & 1 & 1 & 1 & 30 & 15.2 & 28.1 \\
\hline SRSG - 57 & 2 & 5 & 9 & 9 & 9 & 3 & 5 & 3 & 3 & 9 & 3 & 3 & 1 & 5 & 1 & 5 & 5 & 3 & 3 & 3 & 9 & 1 & 1 & 9 & 3 & 3 & 5 & 1 & 5 & 3 & 3 & 3 & 1 & 9 & 33 & 12.3 & 23.9 \\
\hline SRSG - 58 & 2 & 5 & 9 & 9 & 9 & 3 & 5 & 5 & 3 & 9 & 1 & 3 & 1 & 5 & 5 & 5 & 3 & 3 & 5 & 3 & 1 & - & - & 9 & 3 & 5 & 7 & 1 & 3 & 3 & 5 & 3 & 1 & 1 & 39 & 15.4 & 26.3 \\
\hline SRSG - 59 & 2 & 5 & 9 & 9 & 9 & 3 & 3 & 3 & 3 & 9 & 3 & 3 & 1 & 5 & 1 & 3 & 5 & 3 & 3 & 3 & 1 & - & - & 9 & 3 & 3 & 5 & 1 & 5 & 3 & 3 & 3 & 1 & 1 & 31 & 15.3 & 34.8 \\
\hline SRSG - 60 & 2 & 5 & 9 & 9 & 9 & 3 & 5 & 5 & 3 & 9 & 1 & 3 & 1 & 5 & 3 & 3 & 3 & 3 & 3 & 3 & 1 & - & - & 9 & 3 & 5 & 5 & 1 & 5 & 3 & 5 & 3 & 1 & 1 & 23 & 11.1 & 27.7 \\
\hline SRSG - 61 & 2 & 5 & 9 & 9 & 9 & 3 & 3 & 3 & 1 & 9 & 3 & 3 & 1 & 5 & 1 & 3 & 1 & 3 & 3 & 3 & 1 & - & - & 9 & 3 & 5 & 5 & 1 & 5 & 3 & 5 & 3 & 1 & 1 & 38 & 14.0 & 29.9 \\
\hline SRSG - 62 & 2 & 5 & 9 & 9 & 9 & 3 & 5 & 3 & 3 & 9 & 3 & 3 & 5 & 5 & 3 & 3 & 3 & 3 & 3 & 3 & 1 & - & - & 9 & 3 & 3 & 5 & 1 & 5 & 3 & 3 & 3 & 1 & 1 & 25 & 13.6 & 32.1 \\
\hline SRSG - 63 & 2 & 5 & 9 & 9 & 9 & 3 & 5 & 3 & 3 & 9 & 5 & 3 & 1 & 5 & 5 & 5 & 5 & 3 & 3 & 3 & 1 & - & - & 9 & 3 & 5 & 5 & 1 & 3 & 3 & 3 & 2 & 1 & 1 & 39 & 12.6 & 25.5 \\
\hline SRSG - 64 & 2 & 5 & 9 & 9 & 9 & 3 & 5 & 5 & 3 & 9 & 1 & 3 & 5 & 5 & 1 & 5 & 3 & 3 & 5 & 3 & 1 & - & - & 9 & 3 & 3 & 5 & 1 & 3 & 3 & 3 & 2 & 1 & 1 & 27 & 12.7 & 22.2 \\
\hline SRSG - 65 & 2 & 5 & 9 & 9 & 9 & 3 & 5 & 3 & 3 & 9 & 5 & 3 & 1 & 3 & 5 & 1 & 5 & 3 & 3 & 3 & 1 & - & - & 9 & 3 & 3 & 5 & 1 & 5 & 3 & 3 & 3 & 1 & 1 & 31 & 15.6 & 28.7 \\
\hline SRSG - 66 & 2 & 5 & 9 & 9 & 9 & 3 & 5 & 3 & 3 & 9 & 3 & 3 & 1 & 3 & 3 & 3 & 5 & 3 & 3 & 3 & 1 & - & - & 9 & 3 & 5 & 7 & 1 & 5 & 3 & 5 & 2 & 1 & 1 & 33 & 16.5 & 26.8 \\
\hline SRSG - 67 & 2 & 5 & 9 & 9 & 9 & 3 & 3 & 3 & 3 & 9 & 3 & 3 & 1 & 3 & 1 & 1 & 1 & 3 & 3 & 3 & 1 & - & - & 9 & 3 & 3 & 5 & 1 & 3 & 3 & 3 & 3 & 1 & 1 & 29 & 12.9 & 29.0 \\
\hline SRSG - 68 & 2 & 5 & 9 & 9 & 9 & 3 & 5 & 3 & 1 & 9 & 3 & 3 & 5 & 5 & 1 & 5 & 3 & 3 & 3 & 5 & 1 & - & - & 9 & 3 & 5 & 7 & 1 & 3 & 3 & 3 & 3 & 1 & 9 & 28 & 13.5 & 29.1 \\
\hline SRSG - 69 & 2 & 5 & 9 & 9 & 9 & 3 & 5 & 3 & 1 & 9 & 1 & 1 & 1 & 3 & 1 & 3 & 3 & 3 & 3 & 3 & 1 & - & - & 9 & 3 & 5 & 7 & 1 & 3 & 3 & 3 & 2 & 1 & 1 & 26 & 19.8 & 31.3 \\
\hline SRSG - 70 & 2 & 5 & 9 & 9 & 9 & 3 & 3 & 3 & 1 & 9 & 3 & 3 & 1 & 3 & 1 & 3 & 3 & 3 & 3 & 3 & 1 & - & - & 9 & 3 & 3 & 7 & 1 & 5 & 3 & 3 & 3 & 1 & 1 & 28 & 15.4 & 26.7 \\
\hline SRSG - 71 & 2 & 5 & 9 & 9 & 9 & 3 & 5 & 3 & 1 & 9 & 5 & 3 & 1 & 5 & 3 & 3 & 3 & 3 & 3 & 3 & 1 & - & - & 9 & 3 & 5 & 7 & 1 & 3 & 3 & 3 & 3 & 1 & 1 & 26 & 12.9 & 30.5 \\
\hline SRSG - 72 & 2 & 5 & 9 & 9 & 9 & 3 & 3 & 5 & 1 & 9 & 3 & 3 & 1 & 5 & 3 & 3 & 3 & 3 & 3 & 3 & 1 & - & - & 9 & 3 & 5 & 7 & 1 & 3 & 3 & 3 & 2 & 1 & 1 & 42 & 13.5 & 30.0 \\
\hline SRSG - 76 & 2 & 5 & 9 & 9 & 9 & 3 & 5 & 3 & 1 & 9 & 1 & 3 & 1 & 3 & 1 & 3 & 3 & 3 & 3 & 3 & 1 & - & - & 9 & 3 & 3 & 5 & 1 & 3 & 1 & 3 & 2 & 1 & 9 & 25 & 10.3 & 20.3 \\
\hline SRSG - 77 & 2 & 5 & 9 & 9 & 9 & 3 & 5 & 3 & 1 & 9 & 3 & 3 & 1 & 5 & 3 & 3 & 3 & 3 & 3 & 3 & 1 & - & - & 9 & 3 & 3 & 5 & 1 & 3 & 3 & 3 & 3 & 1 & 1 & 33 & 15.5 & 27.3 \\
\hline
\end{tabular}




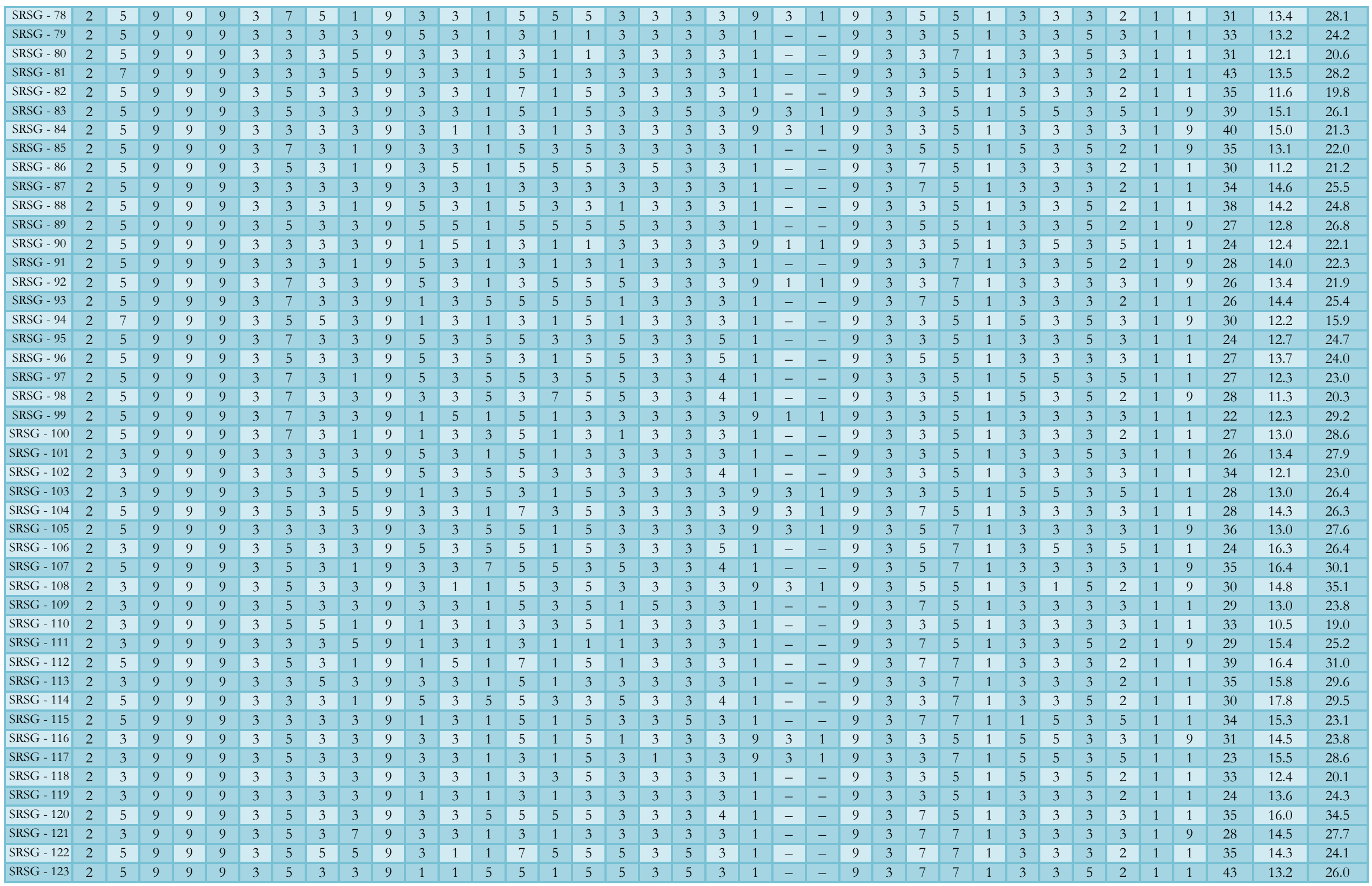




\section{Int.J.Curr.Microbiol.App.Sci (2018) 7(8): 951-959}

\begin{tabular}{|c|c|c|c|c|c|c|c|c|c|c|c|c|c|c|c|c|c|c|c|c|c|c|c|c|c|c|c|c|c|c|c|c|c|c|c|c|c|}
\hline SRSG - 124 & 2 & 5 & 9 & 9 & 9 & 3 & 5 & 3 & 3 & 9 & 1 & 1 & 5 & 5 & 1 & 5 & 3 & 3 & 3 & 3 & 1 & - & - & 9 & 3 & 5 & 7 & 1 & 3 & 3 & 3 & 2 & 1 & 1 & 40 & 12.7 & 26.4 \\
\hline SRSG - 125 & 2 & 5 & 9 & 9 & 9 & 3 & 5 & 3 & 3 & 9 & 5 & 3 & 1 & 5 & 3 & 3 & 5 & 3 & 3 & 5 & 1 & - & - & 9 & 3 & 7 & 7 & 1 & 3 & 3 & 3 & 1 & 1 & 1 & 34 & 14.7 & 28.9 \\
\hline SRSG - 126 & 2 & 3 & 9 & 9 & 9 & 3 & 5 & 3 & 3 & 9 & 1 & 3 & 1 & 5 & 3 & 3 & 5 & 3 & 3 & 3 & 9 & 3 & 1 & 9 & 3 & 7 & 7 & 1 & 1 & 3 & 3 & 3 & 1 & 1 & 33 & 14.9 & 33.1 \\
\hline SRSG - 127 & 2 & 3 & 9 & 9 & 9 & 3 & 3 & 3 & 5 & 9 & 1 & 3 & 1 & 5 & 1 & 3 & 3 & 3 & 3 & 3 & 1 & - & - & 9 & 3 & 3 & 7 & 1 & 3 & 3 & 3 & 3 & 1 & 9 & 33 & 13.6 & 27.8 \\
\hline SRSG - 128 & 2 & 5 & 9 & 9 & 9 & 3 & 3 & 3 & 3 & 9 & 3 & 3 & 5 & 3 & 3 & 3 & 3 & 3 & 3 & 3 & 1 & - & - & 9 & 3 & 3 & 7 & 1 & 3 & 3 & 3 & 2 & 1 & 9 & 32 & 12.5 & 27.6 \\
\hline SRSG - 130 & 2 & 5 & 9 & 9 & 9 & 3 & 3 & 3 & 5 & 9 & 1 & 3 & 1 & 5 & 1 & 3 & 1 & 1 & 5 & 3 & 1 & - & - & 9 & 3 & 3 & 7 & 1 & 3 & 3 & 3 & 2 & 1 & 1 & 28 & 16.9 & 32.0 \\
\hline SRSG - 131 & 2 & 5 & 9 & 9 & 9 & 3 & 3 & 3 & 3 & 9 & 1 & 3 & 1 & 3 & 1 & 3 & 1 & 1 & 5 & 3 & 9 & 1 & 1 & 9 & 3 & 3 & 7 & 1 & 5 & 3 & 3 & 2 & 1 & 1 & 39 & 11.3 & 19.0 \\
\hline SRSG - 132 & 2 & 5 & 9 & 9 & 9 & 3 & 3 & 3 & 3 & 9 & 3 & 3 & 1 & 3 & 1 & 3 & 3 & 3 & 3 & 3 & 1 & - & - & 9 & 3 & 3 & 7 & 1 & 5 & 3 & 3 & 2 & 1 & 1 & 39 & 13.3 & 24.8 \\
\hline SRSG - 133 & 2 & 3 & 9 & 9 & 9 & 3 & 5 & 3 & 5 & 9 & 3 & 3 & 1 & 7 & 3 & 7 & 3 & 3 & 3 & 3 & 9 & 3 & 1 & 9 & 3 & 3 & 7 & 1 & 5 & 5 & 5 & 3 & 1 & 9 & 37 & 9.7 & 19.5 \\
\hline SRSG - 135 & 2 & 5 & 9 & 9 & 9 & 3 & 5 & 3 & 5 & 9 & 5 & 3 & 1 & 7 & 3 & 5 & 3 & 3 & 5 & 3 & 1 & - & - & 9 & 3 & 5 & 7 & 1 & 3 & 1 & 3 & 2 & 1 & 1 & 46 & 12.0 & 23.1 \\
\hline SRSG - 136 & 2 & 3 & 9 & 9 & 9 & 3 & 3 & 3 & 1 & 9 & 1 & 3 & 1 & 5 & 1 & 1 & 1 & 5 & 3 & 3 & 9 & 5 & 1 & 9 & 5 & 3 & 5 & 1 & 3 & 3 & 3 & 2 & 1 & 1 & 26 & 12.3 & 23.0 \\
\hline SRSG - 137 & 2 & 3 & 9 & 9 & 9 & 3 & 3 & 3 & 1 & 9 & 1 & 3 & 1 & 5 & 1 & 1 & 1 & 1 & 3 & 3 & 1 & - & - & 9 & 1 & 3 & 7 & 1 & 3 & 1 & 3 & 2 & 1 & 9 & 40 & 15.3 & 28.3 \\
\hline SRSG - 138 & 2 & 5 & 9 & 9 & 9 & 3 & 3 & 3 & 3 & 9 & 3 & 3 & 1 & 3 & 1 & 3 & 1 & 3 & 3 & 3 & 1 & - & - & 9 & 3 & 3 & 7 & 1 & 3 & 3 & 3 & 2 & 1 & 9 & 29 & 13.5 & 29.5 \\
\hline SRSG - 139 & 2 & 3 & 9 & 9 & 9 & 3 & 3 & 3 & 3 & 9 & 5 & 3 & 5 & 3 & 1 & 1 & 5 & 1 & 3 & 3 & 1 & - & - & 9 & 3 & 7 & 7 & 1 & 3 & 3 & 3 & 3 & 1 & 1 & 27 & 14.6 & 28.1 \\
\hline SRSG - 140 & 2 & 3 & 9 & 9 & 9 & 3 & 3 & 3 & 5 & 9 & 5 & 3 & 1 & 5 & 1 & 3 & 5 & 1 & 3 & 3 & 1 & - & - & 9 & 3 & 7 & 7 & 1 & 3 & 3 & 3 & 1 & 1 & 1 & 23 & 13.0 & 25.1 \\
\hline SRSG - 141 & 2 & 5 & 9 & 9 & 9 & 3 & 5 & 3 & 5 & 9 & 3 & 3 & 1 & 3 & 1 & 5 & 3 & 3 & 5 & 3 & 1 & - & - & 9 & 3 & 7 & 7 & 1 & 5 & 3 & 3 & 3 & 1 & 1 & 39 & 16.4 & 41.6 \\
\hline SRSG - 142 & 2 & 5 & 9 & 9 & 9 & 3 & 5 & 5 & 5 & 9 & 1 & 1 & 5 & 3 & 1 & 5 & 3 & 3 & 3 & 3 & 1 & - & - & 9 & 3 & 5 & 7 & 1 & 3 & 3 & 3 & 3 & 1 & 1 & 40 & 13.2 & 20.4 \\
\hline SRSG - 143 & 2 & 5 & 9 & 9 & 9 & 3 & 5 & 3 & 3 & 9 & 3 & 1 & 5 & 5 & 3 & 5 & 3 & 3 & 3 & 3 & 1 & - & - & 9 & 3 & 5 & 7 & 1 & 3 & 3 & 3 & 3 & 1 & 1 & 24 & 11.9 & 26.5 \\
\hline SRSG - 146 & 2 & 3 & 9 & 9 & 9 & 3 & 3 & 3 & 3 & 9 & 5 & 3 & 1 & 5 & 1 & 5 & 5 & 3 & 3 & 5 & 1 & - & - & 9 & 3 & 5 & 7 & 1 & 5 & 3 & 3 & 2 & 1 & 9 & 15 & 12.8 & 20.7 \\
\hline SRSG - 147 & 2 & 5 & 9 & 9 & 9 & 3 & 5 & 3 & 3 & 9 & 1 & 3 & 1 & 3 & 1 & 1 & 5 & 1 & 3 & 5 & 1 & - & - & 9 & 3 & 5 & 7 & 1 & 3 & 1 & 3 & 1 & 1 & 1 & 38 & 13.3 & 24.2 \\
\hline SRSG - 148 & 2 & 5 & 9 & 9 & 9 & 3 & 5 & 5 & 3 & 9 & 3 & 3 & 1 & 5 & 3 & 3 & 3 & 3 & 5 & 3 & 1 & - & - & 9 & 3 & 3 & 7 & 1 & 3 & 3 & 3 & 2 & 1 & 1 & 40 & 10.8 & 27.3 \\
\hline SRSG - 149 & 2 & 5 & 9 & 9 & 9 & 3 & 5 & 3 & 1 & 9 & 1 & 3 & 1 & 5 & 1 & 5 & 5 & 3 & 3 & 3 & 1 & - & - & 9 & 3 & 5 & 9 & 1 & 1 & 3 & 3 & 3 & 1 & 1 & 32 & 13.9 & 25.6 \\
\hline SRSG - 150 & 2 & 3 & 9 & 9 & 9 & 3 & 3 & 3 & 3 & 9 & 3 & 3 & 1 & 3 & 1 & 3 & 3 & 3 & 5 & 3 & 1 & - & - & 9 & 3 & 7 & 7 & 1 & 1 & 1 & 3 & 1 & 1 & 1 & 28 & 17.7 & 28.6 \\
\hline SRSG - 151 & 2 & 5 & 9 & 9 & 9 & 3 & 5 & 3 & 3 & 9 & 5 & 3 & 5 & 7 & 5 & 5 & 5 & 3 & 3 & 3 & 1 & - & - & 9 & 3 & 5 & 7 & 1 & 3 & 3 & 3 & 3 & 1 & 1 & 33 & 18.7 & 28.7 \\
\hline SRSG - 152 & 2 & 5 & 9 & 9 & 9 & 3 & 3 & 3 & 3 & 9 & 5 & 3 & 5 & 5 & 1 & 3 & 5 & 3 & 5 & 3 & 1 & - & - & 9 & 3 & 7 & 7 & 1 & 3 & 3 & 5 & 2 & 1 & 1 & 35 & 13.7 & 26.4 \\
\hline SRSG - 153 & 2 & 3 & 9 & 9 & 9 & 3 & 5 & 5 & 1 & 9 & 3 & 3 & 1 & 5 & 5 & 5 & 3 & 3 & 5 & 3 & 1 & - & - & 9 & 3 & 7 & 7 & 1 & 3 & 3 & 3 & 3 & 1 & 1 & 36 & 14.4 & 24.2 \\
\hline SRSG - 154 & 2 & 5 & 9 & 9 & 9 & 3 & 5 & 5 & 1 & 9 & 3 & 3 & 1 & 5 & 5 & 1 & 3 & 3 & 3 & 3 & 1 & - & - & 9 & 3 & 7 & 7 & 1 & 1 & 3 & 3 & 2 & 1 & 9 & 34 & 17.3 & 24.8 \\
\hline SRSG - 155 & 2 & 5 & 9 & 9 & 9 & 3 & 5 & 3 & 1 & 9 & 3 & 3 & 5 & 5 & 3 & 5 & 3 & 3 & 3 & 3 & 1 & - & - & 9 & 3 & 7 & 7 & 1 & 5 & 3 & 3 & 3 & 1 & 1 & 34 & 15.6 & 34.5 \\
\hline SRSG - 156 & 2 & 5 & 9 & 9 & 9 & 3 & 7 & 3 & 7 & 9 & 5 & 3 & 5 & 5 & 5 & 5 & 5 & 3 & 3 & 3 & 1 & - & - & 9 & 3 & 7 & 7 & 1 & 3 & 3 & 3 & 3 & 1 & 1 & 42 & 16.2 & 22.0 \\
\hline SRSG - 157 & 2 & 5 & 9 & 9 & 9 & 3 & 7 & 3 & 3 & 9 & 3 & 3 & 5 & 5 & 5 & 7 & 5 & 3 & 3 & 5 & 1 & - & - & 9 & 3 & 7 & 7 & 1 & 5 & 5 & 3 & 5 & 1 & 9 & 44 & 10.9 & 23.3 \\
\hline SRSG - 158 & 2 & 5 & 9 & 9 & 9 & 3 & 5 & 5 & 3 & 9 & 3 & 3 & 1 & 5 & 3 & 3 & 5 & 3 & 5 & 3 & 1 & - & - & 9 & 3 & 7 & 7 & 1 & 7 & 5 & 5 & 5 & 1 & 1 & 35 & 18.2 & 26.3 \\
\hline SRSG - 159 & 2 & 5 & 9 & 9 & 9 & 3 & 5 & 5 & 5 & 9 & 3 & 3 & 1 & 5 & 3 & 7 & 3 & 3 & 5 & 3 & 1 & - & - & 9 & 3 & 3 & 7 & 1 & 3 & 5 & 3 & 5 & 1 & 1 & 38 & 15.7 & 28.5 \\
\hline SRSG - 160 & 2 & 5 & 9 & 9 & 9 & 3 & 5 & 5 & 5 & 9 & 3 & 3 & 1 & 7 & 1 & 5 & 3 & 3 & 5 & 3 & 1 & - & - & 9 & 3 & 3 & 7 & 1 & 3 & 5 & 3 & 5 & 1 & 1 & 32 & 15.9 & 25.0 \\
\hline
\end{tabular}


Among the 160 rice genotypes, 52 had thin type of stem, 92 with medium type and 16 genotypes were thick type category. Stem: length was very short in 83 genotype, short in 43, medium in 29 and long in five genotypes. Similar results obtained by Shrivastava et al., (2015), Rajanna et al., (2011).

Twenty genotypes were very short, 66 genotypes were short, 67 genotypes with medium and seven genotypes showed long panicle length. Out of 160 genotypes, 30 were having erect attitude of blade of flag leaf, 91 semi-erect attitude of blade of flag leaf, 39 horizontal attitude of blade of flag leaf. Nine were straight curvature, 146 were having semi-straight curvature and five genotypes showed deflexed curvature of panicle. 129 had few, 29 had medium and two genotypes showed many number of panicles per plant. Among 160 genotypes, one had yellow, 136 with brown colour, six had red and 17 genotypes with purple coloured lemme tip. Among 160 genotypes, awn was absent in 127 genotypes and present in 33 genotypes. Out of 33 awned genotypes, 14 were very short type, medium type observed in 14 genotypes and other five genotypes were long type of panicle. Out of 33 genotypes having awns, 31 had their awns on tip only and in two genotypes awns were distributed all over the length of panicles. Two genotypes showed erect type of attitude of panicle branches, 153 genotypes were of erect to semi-erect and semi-erect in five genotypes. Out of 160 genotypes, 89 genotypes were partly exerted, 43 were of mostly exerted type and 28 genotypes showed exerted type of panicle. Similar results obtained by Shrivastava et al., (2015), Rajanna et al., (2011).

1000 grains weight was very low $(<15 \mathrm{~g})$ in 10 genotypes, low (15-20 g) in 98, medium (21-25 g) in 49 and high (26-30) in three genotypes. Decorticated grain length was short in 11, medium in 126 and long in 23 genotypes. Decorticated grain width was narrow $(<2.0 \mathrm{~mm})$ in 125 and medium $(2.0-$ $2.5 \mathrm{~mm})$ in 35 genotypes. Seven genotypes showed short slender, 50 genotypes had short bold, 82 genotypes had medium slender and 21 genotypes had long slender decorticated grain shape. Hundred and twenty-five genotypes grains were devoid of aroma and other 35 genotypes had aroma in their grains. Similar results obtained by Shrivastava et al., (2015), Rajanna et al., (2011). SPAD values had low $(<30)$ in 58, medium $(30-40)$ in 91 and good (>40) in 11 genotypes. Among 160 genotypes the iron content ranges from 9.7 to $19.8 \mathrm{mg} / \mathrm{kg}$ and zinc content from 15.9 to 41.9 $\mathrm{mg} / \mathrm{kg}$. These results were in conformity with results of Maddeppa et al., (2017).

In conclusion, characterization is the most basic and important step in the process of evaluation, cataloguing of variety and protection against illegal utilization. Morphological (DUS) characters are useful to explore the relatedness, variations and distinctiveness amongst varieties.

\section{References}

Maddeppa, M., Surendra, P., Hanamaratti, N. G., Jogi, M., Sathisha, T. N. and Hundekar. R., 2016, Genetic variability for yield and yield attributing traits in $\mathrm{F}_{3}$ generation of rice (Oryza sativa L.). Res Environ Life Sci., 9(1) 24-28.

Mishra, S. K., Sharma, B., Dasgupta, S. K. and Kabillon, R., 1996, Electrophoretic variation for seed protein of pea. Seed Res., 24(2): 8 8-92.

Rajanna, C. M., Narayanaswamy, R. B., Choudhury, M. M., Jaume, A., Silva, T, D. and Prasad R., 2011, Characterization of rice (Oryza sativa) hybrids and their parental lines based on seed, seedling and plant morphological traits. Seed sci. biotech., 5(1): 36-41.

Shrivastava, A., Koutu, G. K., Mishra, D. K. 
and Singh, S. K., 2015, Characterization Pl. Arch., 15(1): 397- 403. of JNTP lines of rice (Oryza sativa L.).

\section{How to cite this article:}

Komala, N.T. and Gurumurthy, R. 2018. DUS Characterization and Biofortification of Rice Genotypes Derived from Swarna x Ranbir Basmati Cross. Int.J.Curr.Microbiol.App.Sci. 7(08): 951-959. doi: https://doi.org/10.20546/ijcmas.2018.708.108 